\title{
BONPLANDIA
}

\section{Estudios ecológicos en los campos del sur de Misiones II.}

\section{La repoblación vegetal de los tacurúes.}

\author{
por RAUL MARTINEZ-CROVETTO
}

Tacurú es el nombre con que en el nordeste del país se conocen las comejeneras o termiteros, nidos de la termita Conitermes similis (Hag.). Tienen éstos forma de columna cónica, que puede llegar a dos metros de altura y alcanzar, en la base, un metro de diámetro. Su superficie terrosa es muy dura y compacta, sólo penetrable a fuerza de pico, presentando el mismo color rojo del suelo con que fueron construidos.

$\mathrm{Su}$ estructura interior, vista en sección transversal, muestra dos sectores claramente delimitados: en primer lugar, la parte periférica formada por un conjunto de estrechas galerías y paredes gruesas, todo dispuesto en forma bastante irregular; en segundo lugar, la porción interior, donde las galerías son mayores, más regulares y las paredes delgadas. Si se observa un tacurú en sección longitudinal, aparecen como los pisos de un edificio.

Los tacurúes son muy comunes en todo el sur de Misiones, en el sector fitogeográfico Austro-occidental, preferentemente en las comunidades campestres con predominio de Aristida jubata y de Andropogon lateralis. A veces, en algunos lugares son tan abundantes que da la impresión de que se hubieran plantado postes con cierta regularidad.

En las últimas décadas, con el auge de la ganadería y de la agricultura en la mencionada región, los pobladores se han visto en la necesidad de combatirlos y, hoy día, los campos se han ido librando de su molesta presencia, hasta quedar ésta reducida a la vera de los caminos o a lugares poco accesibles.

Cuando las termitas son destruidas o el nido abandonado, los agentes climáticos van ablandando la tierra $y$, al fin de un cierto tiempo, variable según la intensidad y frecuencia de las lluvias, 
Ciadro $n^{\circ}$ 1.- Tacurúes en proceso de repoblación en el AndropogonetoExonopetum (parceias de $1 \mathrm{~m}^{2}$ ) (15-I-1960).

Número del censo

Cobertura $(\%)$

H Paspalum notatum

C Eupatorium subhastaium

$\mathrm{T}$ Gerardia communis

H Desmodium canum

H Peltodon longipes

$\mathrm{H}$ Arachis hagenbeckii

C Sida rhombifolia

$\mathrm{H}$ Elephantopus mollis

$\mathrm{H}$ Iponoea descolei

$\mathrm{H}$ Ipomoea kunthiana

$\mathrm{H}$ Andropogon lateralis

C Scoparia hassleriana

$\mathrm{T}$ Conyza bonariensis

C Heimia salicifolia

$\mathrm{T}$ Conyza blakei

H Manihot hunzikeriana

$\begin{array}{rrrrrrrrrrrr}1 & 2 & 3 & 4 & 5 & 6 & 7 & 8 & 9 & 10 & \mathrm{p} & \mathrm{C} \\ 90 & 95 & 95 & 90 & 95 & 85 & 85 & 90 & 85 & 95 & & \end{array}$

$\begin{array}{llllllllllll}5.5 & 5.5 & 5.5 & 4.5 & 5.5 & 5.5 & 4.5 & 5.5 & 5.5 & 4.5 & 10 & \mathrm{~V}\end{array}$

$1.21 .2+.11 .2+.1+.11 .1 .+11.29 \mathrm{~V}$

$+.1 \cdot 1.1+.1+.1+.1+.1+.1 .1 .18$ IV

$.+.1+.1$. $+.1+.1$. +.15 III

$1.1 \cdot+.1 \cdot+.1 .+.14$ II

$+1 .+.1+.1 .3$ II

$+1.1 .1+.1 .3$ II

. $+1.1+1.1+3$ II

. . $+1+.1 .2 .2$. 2

. . . $.1 .+1.1 .2$ i

. $\quad .11 .1 . \quad . \quad 2 \quad 2$ I

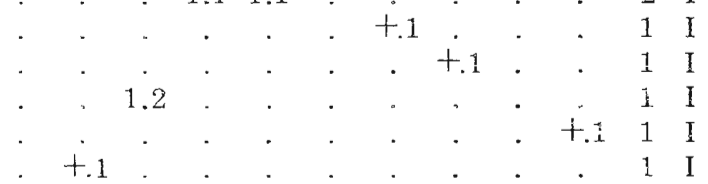

Cuadro n².- Tacurúes en proceso de repoblación en el AristidetoPaspaletum (parcelas de $1 \mathrm{~m}^{2}$ ) (15-I-1960).

Número del censo

Cobertura $(\%)$

H Paspalum notatum

C Eupatorium subhastum

$\mathrm{T}$ Gerardia communis

If Aristida jubata

H Desmodium canum

$\mathrm{T}$ Conyza blakei

$\mathrm{H}$ Peltodon longipes

H Ocimum nudicaule

$\mathrm{H}$ Ipomoea kunthiana

H Euphorbia hebegyne

G Euphorbia papillosa

$\mathrm{H}$ Arachis hagenbeckii

C Tibouchina gracilis

H Ipomoea descolei

G Dichondra sericea

H Melancium campestre $\begin{array}{llllllllllll}1 & 2 & 3 & 4 & 5 & 6 & 7 & 8 & 9 & 10 & \mathrm{P} & \mathrm{C}\end{array}$

$\begin{array}{llllllllll}90 & 85 & 75 & 75 & 85 & 75 & 80 & 85 & 95 & 85\end{array}$

$\begin{array}{lllllllllllll}5.5 & 5.5 & 5.5 & 3.5 & 4.5 & 2.4 & 3.5 & 3.5 & 5.5 & 4.5 & 10 & \text { V }\end{array}$

+.11 .2 . $1.2+.21 .2+.1$. +.17 IV

. $+.1 .+.1+.1$. +.14 II

. . $1.2+.21 .2 . \quad+.1 .4$ II

$.+1 .+1+.1 .1 .14$ II

$+.12 .1 .+.1 .3 \mathrm{n}$

$+1 .+.1+.1 .3$. 3

$. \quad .+1+1.2 .21$

$1.1 .5 . \quad . \quad .11$

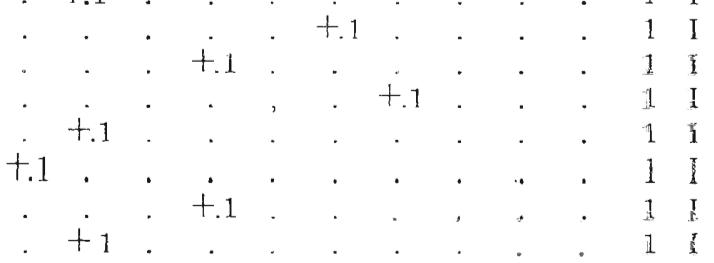


Cuadro $n^{\circ}$ 3.- Comparación entre las especies colonizadoras con mayor grado de constancia en los tacurúes de ambas asociaciones.

Asociación
Paspalum notatum
Eupatorium subhastatum
Gerardia communis
Desmodium canum
Peltodon longipes
Andropogon lateralis
Aristida jubata
Conyza blakei
Arachis hagenbeckii
Sida rhombifolia
Ipomoea descolei
ipomoea kunthiana
Ccimum nudicaule
Elephantopus mollis

Andropogoneto Aristideto

Paspalum notatum

Eupatorium subhastatum

$\mathrm{V}$

V IV

IV II

Gerardia communis

III II

Peltodon longipes

II II

Andropogon lateralis

II -

Aristida jubato

- II

Conyza blakei

I II

Arachis hagenbecki

II I

Sida rhombifolia

ipomoea kunthiana

Elephantopus mollis

V
IV
II
II
II
II
II
I
I
I
II
-

se derrumban, quedando en ei sitio montículos o túmulos. Estos tienen forma de casquete esférico un tanto irregular y pueden alcanzar hasta 30 o 40 centímetros de altura por varios metros de diámetro, generalmente 2 o 3 .

Al cesar toda la influencia de las termitas, se inicia la repoblación vegetal del suelo que ha quedado descubierto. Comienza ésta en dirección centrípeta y se caracteriza por la invasión de Paspalum notatum que, gracias a sus rizomas estoloniformes resistentes al pastoreo y a las quemazones, se posesiona del terreno hasta llegar a cubrirlo totalmente. Junto con esta gramínea, aparece la caméfita Eupatorium subhastatum, también de alta constancia. Le siguen Desmodium canum, Ipomoea descolei e Ipomoea kunthiana, que penetran aesde el exterior merced a sus tallos o ramas rastreras. Lel mismo modo se observa el ingreso al área de Peltodon longipes y de Melancium campestre.

Para estajolecer la constancia de ias especies en tacurúes en proceso de repoblación, se eligió un terreno próximo al Aeroclub cie Posadas, donde, en una extensión de varias hectáreas, su propietario se había dedicado a combatir las termitas. La parte alta estaba ocupada por el Aristideto-Paspaletum y el bajo por el Andropogoneto-Axonopetum, ambas asociaciones en proceso de destrucción por las quemazones y el pastoreo bastante intenso. En cada una de sus respectivas áreas, se efectuaron 10 relevamientos de $1 \mathrm{~m}^{2}$ de superficie, según $e_{\alpha}^{\prime}$ sistema abreviado de BraunBlanquet, ubicancic cada parcela en un tacurú en repoblación diferente. ios resultados se dan en los cuadros. 


\section{Conclusiones}

1. Cuando los nidos de la termita Conitermes similis son destruidos, la repoblación vegetal se inicia con la invasión lateral de Paspalum nctatum

2. Cuando dicha gramínea ha cubierto toda el área, aparece Eupatorium subhastatum, que alcanza alto grado de presencia.

3. En el total de parcelas censadas se detectó la existencia de 23 especies diferentes.

4. La mayoría de éstas, con la excepción de Andropogon lateralis y Elephantopodus mollis, características del Andropogoneto, y de Aristida jubata y Ocimum nudicale, características del Aristideto, son especies comunes a ambas asociaciones.

5. En consecuencia, el proceso de repoblación vegetal de los tacurúes, por lo menos bajo las condiciones de alteración debidas a las quemazones y el pastoreo, es similar en las dos comunidades analizadas.

\section{Summary}

This study is about the process of vegetal repopulate on uncovered areas, coming from the destruction of the termite Conitermes similis nests. This isoptheia mainly nests in the Andropoyoneto-Axonopetum and Aristideto-Paspaletum soils. 